Trinity University

Digital Commons @ Trinity

Psychology Faculty Research

Psychology Department

6-2015

\title{
Distracted by Cues for Suppressed Memories
}

\author{
Paula T. Hertel \\ TrinityUniversity, phertel@trinity.edu \\ Jeffrey A. Hayes \\ Trinity University, jhayes1@trinity.edu
}

Follow this and additional works at: https://digitalcommons.trinity.edu/psych_faculty

Part of the Psychology Commons

Publication Details

Psychological Science

\section{Repository Citation}

Hertel, P.T., \& Hayes, J.A. (2015). Distracted by cues for suppressed memories. Psychological Science, 26(6), 775-783. doi: 10.1177/0956797615570711

This Post-Print is brought to you for free and open access by the Psychology Department at Digital Commons @ Trinity. It has been accepted for inclusion in Psychology Faculty Research by an authorized administrator of Digital Commons@ Trinity. For more information, please contact jcostanz@trinity.edu. 


\section{Distracted by Cues for Suppressed Memories \\ Paula T. Hertel and Jeffrey A. Hayes \\ Trinity University}

Words: 2046 (introduction, introduction to Exp 2, discussion, acknowledgement)

Tables: 0

Figures: 3

Author Note

Paula T. Hertel and Jeffrey A. Hayes, Department of Psychology, Trinity University.

Correspondence concerning this article should be addressed to Paula Hertel, Department of Psychology, Trinity University, San Antonio, TX 78212; phertel@ trinity.edu. 


\begin{abstract}
We examined the potential cost of practicing suppression of negative thoughts for subsequent performance in an unrelated task. Cues for previously suppressed and baseline responses in a think/no-think procedure were displayed as irrelevant flankers for neutral words to be judged for emotional valence. These critical flankers were homographs with one negative meaning denoted by their paired responses during learning. Suppression cues as flankers delayed responding to the targets, compared to baseline cues and new negative homographs, but only following directsuppression instructions and not when benign substitutes had been provided to aid suppression. On the final recall test, suppression-induced forgetting (SIF) following direct suppression and the flanker task was positively correlated with the flanker effect. Experiment 2 replicated these findings. Finally, valence ratings of neutral targets were influenced by the valence of the flankers but not by the prior role of the negative flankers.
\end{abstract}

Keywords: suppression, inhibition, TNT, distraction, forgetting 


\section{Distracted by Cues for Suppressed Memories}

According to some perspectives, the deliberate suppression of negative thoughts and memories is ill advised. For example, the suppression of intrusive memories and flashbacks associated with Post-Traumatic Stress Disorder seems to exacerbate the problem (see the commentary by Holmes, Moulds, \& Kavanagh, 2007). On the other hand, the benefits of suppression for forgetting in mundane situations have been clearly enumerated (Bjork, 1989) and potentially extend to some clinical contexts (Stephens, Braid, \& Hertel, 2014). A main element of this debate concerns the viability of thought suppression as a mechanism for forgetting. Wegner (1994) argued that such efforts are inevitably followed by rebound of the suppressed thought. Yet Anderson and Huddleston (2012) reviewed scores of experiments showing convincing evidence of suppression-induced forgetting (SIF) by participants asked to recall suppressed and unsuppressed words and images later on.

Although demonstrations of SIF in deliberate recall tasks have been important theoretically, when people suppress negative thoughts and memories in everyday life they rarely try to remember them later. The more interesting evidence of SIF, in our view, is found in tasks involving indirect measures of memory (e.g., Gagnepain, Henson, \& Anderson, 2014; Kim \& Yi, 2013). For example, Hertel, Large, Stuck, and Levy (2012) distributed cues for previously suppressed or baseline (unsuppressed) response words throughout a subsequent free-association task, disguised as a separate experiment. Fewer suppressed words were produced. Moreover, SIF generalized beyond the specific negative response words. The cues were homographs with both negative and benign meanings, and fewer associative responses denoting the negative meanings of the cues were produced in response to suppression cues than in response to baseline cues.

In thinking about the evidence for indirect effects of suppression, we wondered whether other indirect measures might reveal costs of suppression instead of the frequently demonstrated 
benefits - an homage to Wegner's notion of the ironic effects of control. Clearly, sustained efforts to control the focus of attention are required during the many suppression trials in the think/no-think (TNT) paradigm invented by Anderson and Green (2001). After learning to produce response words upon presentation of their cues, participants stare at a subset of the cues for at least $3 \mathrm{~s}$ each, trying hard not to allow the response word to come to mind, and this procedure is repeated as many as 18 times per cue. Indeed, the greater the activation of prefrontal cortical areas associated with attentional control during those "no-think" trials, the larger the SIF effect in subsequent cued recall (Anderson et al., 2004; Depue, Curran, \& Banich, 2007). Similarly, we reasoned that the suppression cues themselves might invite attention on other occasions, more so than if the responses to those cues had not been suppressed. In a "real-world" example, someone might repeatedly be determined not to think about the breakup of a longstanding friendship when conversing with the friend's colleague, but the colleague's presence on subsequent occasions subtly seems to distract attention and to serve as a cue for stopping one's thoughts.

To capture this phenomenon, we used a version of the flanker paradigm (Eriksen \& Eriksen, 1974), disguised as a separate experiment performed to investigate ratings of emotional valence. A few negative and positive and many more neutral words were individually centered on the monitor and flanked above and below with another word (a flanker). Many flankers were homographs with possible meanings ranging from very negative to very positive. The critical homographs for our main purpose, however, were the negative homographs that had appeared as baseline or suppression cues in the previous TNT "experiment" (as well as unexperienced negative homographs, fully rotated). We predicted that participants would be delayed in rating the neutral targets flanked by suppression cues, recently functioning as cues for stopping all 
thoughts. Importantly, we predicted that this distraction effect would depend on direct suppression and not just the recency of exposure to the cues.

To control for recency, we included substitution as an alternative condition to direct suppression. A frequently replicated effect in the literature on SIF is that the effect is stronger when experimenter-provided substitutes are brought to mind during suppression attempts (e.g., porcelain-goblet in place of porcelain-doll) than when participants are given no particular instructions for how to suppress thoughts of the response words (e.g., Hertel \& Calcaterra, 2005). Few comparisons of SIF have been made between conditions with experimenter-provided substitutes and those that discourage participant-generated substitutes (direct suppression). Benoit and Anderson (2012), however, showed that the processes underlying SIF aided by experimenter-provided substitutes are indeed quite different from those corresponding to directsuppression attempts. In direct suppression, activation of the right dorsolateral prefrontal cortex is associated with the down-regulation of hippocampal or neocortical regions. In contrast, participant-generated thought substitution activates both the left inferior frontal gyrus and hippocampal areas (Benoit \& Anderson, 2012; see Anderson \& Hanslmayr, 2014). Thus, the substitution method provides a clear comparison to direct suppression. Substitution instructions redirect the focus of "mental" attention (to the benign substitute, in this case) while still requiring visual attention to the cues to a similar extent as required by the direct suppression method and with equal frequency and recency with respect to the flanker task.

Another purpose in using a substitution condition was to show the potential consequences of redefining the cue. After a few interesting conversations with the colleague in our previous example, the topic of those conversations might help us to not think about the former friend, and seeing the colleague in meetings no longer distracts. He is no longer associated primarily with 
something that we don't want to think about. In Experiment 1, we cued with homographs and provided benign substitutes for the negative response words to be suppressed, reasoning that the meaning of the suppression cues would change while the perceptual stimuli remain the same. We evaluated this prediction by examining the ratings of neutral targets surrounded by suppression cues in the substitution versus the direct-suppression condition. Later, following the flanker task, we tested recall of the negative responses to assess SIF in both conditions following an intervening task, with the aim of extending the ecological validity of the recall test.

\section{Experiment 1}

\section{Method}

Materials. Although only 24 cues were actually encountered prior to the flanker task, a total of 32 cues were needed for the learning, TNT, and recall phases of the experiment: 24 homographs with at least one negative and one benign meaning and 8 non-homographs with benign meanings. Each cue was paired with a respond word, described next. The flanker task required 56 targets to be judged for their valence and 32 new flanker words (in addition to the 24 negative homographs prepared for the TNT phase). Each target was presented with the flanking word displayed both above and below it. All types of trials are represented in Figure 1.

Learning and TNT materials. Twenty-four homographic cues were assigned both a related negative response word and a related benign substitute (e.g. vent-frustration and ventwindow). Prior to the experiment 68 students rated all cue-response and cue-substitute pairs for either imagery or emotional valence (each on a 7-pt scale). Those pilot ratings, together with forward associative strengths (Nelson, McEvoy, \& Schreiber, 1998), cue, response, and substitute frequencies (Kucera \& Francis, 1967), numbers of letters, and the part of speech defined for the cue by each response word were used to construct three balanced sets of 8 triplets 


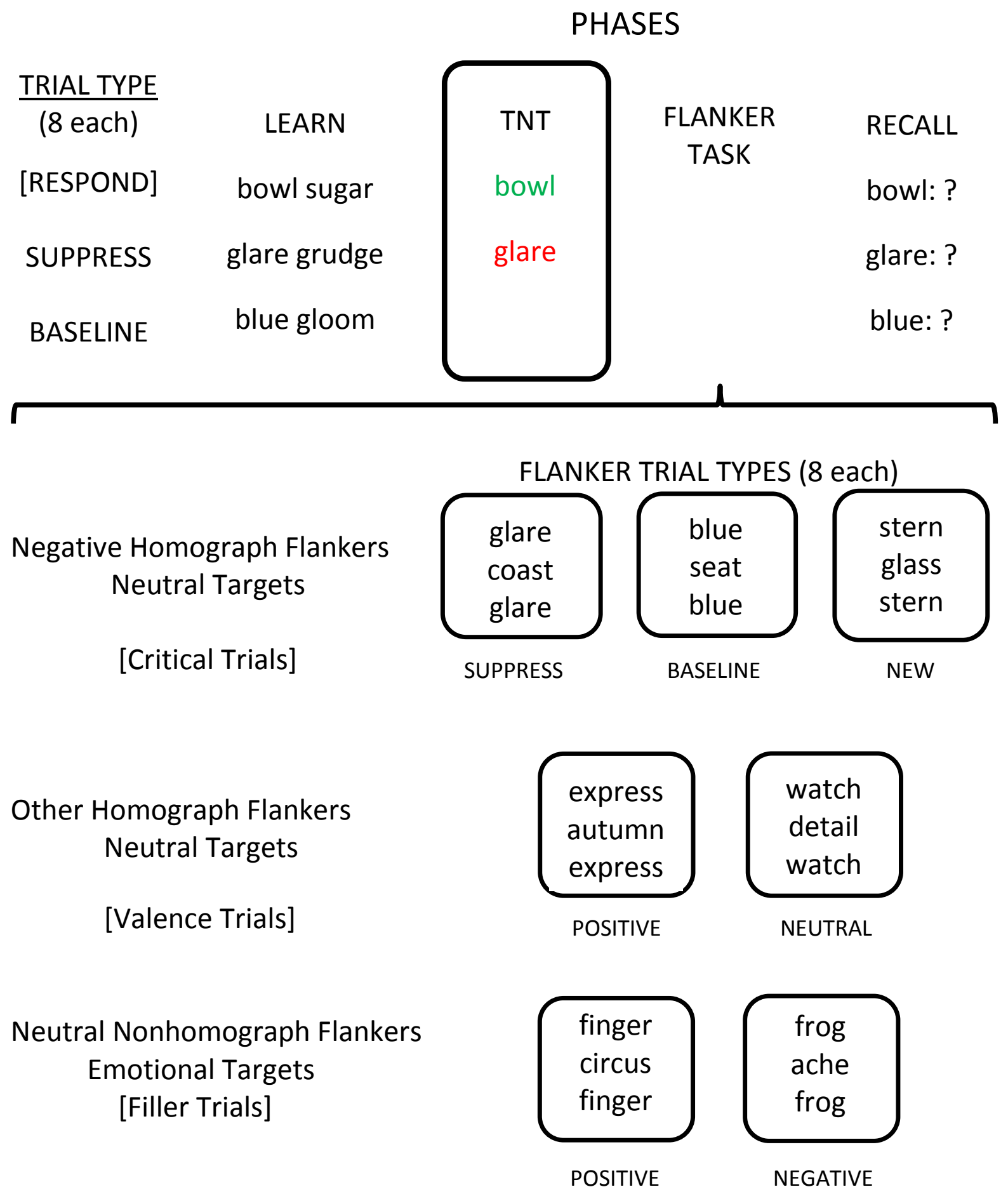

Figure 1. Schematic representation of the four main phases of the experiment (learn, TNT, flanker task, recall test) and the types of trials on the flanker test. The 24 critical trials consisted of negative homographic flankers and neutral targets ( 8 flankers having served as cues for suppression, 8 as cues from baseline pairs not presented in the TNT phase, and 8 new cues from pairs not seen in prior phases; all fully counterbalanced). To assess flanker valence differences, 16 trials presented neutral targets flanked by either positive or neutral homographs (to compare 
to the 8 new negative homographs from critical trials). To make the rating task meaningful to the participants, 16 filler trials contained either positive or negative targets with neutral nonhomographic flankers. 
each (the critical sets). The role assigned to the cues in each set rotated across the conditions of the TNT phase-suppression, baseline (learned but not seen in the TNT phase), and new negative (neither learned nor seen in the TNT phase)—in a counterbalanced fashion within each condition of instruction (direct suppression or substitution). In addition, eight non-homograph cues with benign response words (e.g., butter-pancakes) served as Respond items during the TNT phase. They were initially learned, practiced during the TNT phase, and cued during recall, but they did not appear in the flanker task.

Additional words for the flanker task. Of the 56 target words in the flanker task, 40 were neutral to slightly positive words with valence ratings within the range of 5.0-6.2 on the 9-pt scale used in the Affective Norms for English Words (ANEW; Bradley \& Lang, 1999). Each neutral target was paired with a homograph flanker, not related in meaning (according to our judgment) but with the same on-screen word length. These pairs were constructed in five sets of 8 , including flankers identified as the three critical sets of 8 negative homographic cues from the learning phase, 8 new positive homographs, and 8 new neutral homographs. The targets in these five sets were balanced on word length, valence rating (ANEW), arousal rating (ANEW), frequency, and parts of speech.

To vary the actual valence of the targets to be rated for emotionality, the remaining 16 targets were selected from positive and negative words in ANEW. The mean valence rating was 7.1 for the 8 positive targets and 2.1 for the 8 negative targets. These emotional targets were paired with 16 neutral, non-homographic flanker words, and the appropriate balancing was achieved.

Fillers and buffers. Eight additional benign pairs served as buffers or fillers in the learning and TNT phases. Another eight benign pairs were presented at the beginning or end of 
the flanker task. All elements of all these pairs were emotionally neutral or slightly positive nonhomographs.

Participants and design. Based on cell sizes employed in other TNT experiments in our lab and elsewhere, we planned to recruit 21 participants for each condition of Instruction Method (direct suppression and substitution); multiples of 3 were required for counterbalancing the sets that rotated across the status of the cue (suppression, baseline, and new homographs). A total of 50 Trinity University undergraduates (29 female and 21 male) seeking course-required credit volunteered for what we described as two experiments—one that required the learning of word pairs and another shorter experiment to assess the emotional value of words. Eight students' data (five from the direct-suppression condition and three from the substitution condition) were set aside due to noncompliance with suppression instructions in the TNT phase (i.e. the reported occasional and deliberate use of strategies for rehearsing response words assigned to be suppressed). When noncompliance was identified we recruited a replacement. The final sample therefore included 21 participants (12 women) in each instruction condition, with full counterbalancing of materials. Age ranged from 18 to 21 in both experiments.

Procedure. All programming for the experiment was done with SuperLab Pro (Version 5.0; Cedrus Corporation, San Pedro, CA). All trials in all phases began with a 200-ms orientation display (two crosses separated by 10 spaces horizontally) and ended with a 500-ms ISI. All events were centered on the screen and displayed in regular Arial 20-pt font on white background; event color was black unless otherwise noted.

Learning phase. One third of the trials consisted of the pairs scheduled for responding during the TNT phase, and two thirds consisted of pairs from two critical sets. Each pair was displayed for $5 \mathrm{~s}$, during which time participants were asked to create a mental image for the pair 
while learning it for an immediate test. Each pair was followed by a rating scale ranging from "not at all vivid" to "extremely vivid," separated by numerals 1 to 7 . Participants typed a number to rate the vividness of the image. The order of the pairs was randomized anew within blocks of three, each block containing a pair scheduled for response, suppression, or nonpresentation (baseline) in the subsequent TNT phase. After presenting all pairs, we tested learning by displaying the cues alone in the same randomized-block ordering and by dropping cues when the response was correctly produced. Each cue was presented for $5.2 \mathrm{~s}$ or until a correct response (spoken aloud and keyed by the experimenter), and then the correct response word was presented in blue for $2 \mathrm{~s}$.

TNT phase. The participants first completed a brief practice task using the filler pairs. During practice and the full TNT phase, a green cue signaled the participant to recall the associated response. A red cue signaled participants to not think about the associated response, while paying full attention to the cue throughout the display. All cues were presented for $3 \mathrm{~s}$, or less in the case of correctly produced responses to green cues. Instructions in both conditions identically and repeatedly emphasized the importance of preventing the response word from coming to mind in any form while looking at the cue. In both conditions, erroneous mention of the response to a red cue was followed by a string of large red Xs.

In the direct-suppression condition we urged participants not to replace the response to red cues with any other thoughts. This instruction was delivered on several occasions before and after the practice task as the experimenter and participant talked through an assessment. In the substitution condition, we gave the participants a substitute to use during the practice task, and then the full set of eight substitutes were presented with their cues (e.g., vent-window) to be studied briefly prior to the main TNT phase. Participants were asked to respond with the 
substitute when cues were presented in red, and the substitute was displayed for $500 \mathrm{~ms}$ following each 3-s cue display.

Order of cues in the main TNT phase was randomized within four blocks of six items; each block contained two cues for responding (in green), two fillers (in green), and two red cues (from one of the rotated critical sets). All blocks were presented 16 times, with a break occurring half-way through. Afterward, participants filled out a strategy questionnaire to assess their level of compliance with suppression instructions, their use of substitutes (see Hertel \& Calcaterra, 2005), and the frequency of difficulty in suppression. The importance of this questionnaire was exaggerated, so that participants might believe that it assessed their ability to not think about words they had learned, in continuation of the "two experiment" cover story.

Flanker task. In the so-called second experiment of the session, participants were asked to complete a series of trials in which they rated the emotional value of the middle word of a display of three words, aligned vertically. (According to the cover story, other participants would be asked to rate the word presented on the top and bottom.) Participants were told that this flanker word was unimportant; their task was to rate the middle word as quickly and accurately as they could on a scale from 1 to 9 , where 1 meant strongly negative and 9 meant strongly positive. When they had a rating in mind, they pressed the space bar to reveal the rating scale, at which point they could type the chosen number. We included the spacebar tap as a step to make times less noisy than if the participants needed to search for number keys during the main display.

The order of the 56 flanker pairs was randomized within eight blocks of 7 events, one from each type illustrated in Figure 1. The experimenter watched the completion of a practice 
block of four buffer pairs to ensure understanding and compliance before moving behind a screen.

Final recall. Described as a surprise memory test, the final task requested recall of all the initially learned response words, regardless of prior instruction during the TNT phase. We told the participants that the preceding task of rating emotion had served to fill the interval before this test could be given. All 24 cues from the critical sets of homographs, plus the 8 cues for responding during the TNT phase, were presented again in the same randomized block ordering as initially used. Each cue appeared for $4 \mathrm{~s}$. While emphasizing the importance of recalling the initial response word, we told participants that they could provide two words if two should come to mind. If they reported two words, they identified the original word from the learning phase.

\section{Results}

Flanker effects. Our primary dependent measure was the time to tap the spacebar in order to rate valence. We were also interested in the rating itself, both as a measure of whether participants stayed on task (by examining ratings of new negative, neutral, and positive targets) and as a measure of interference as a function of both the valence of and prior experience with the flankers. In all analyses, the significance level was .05. Huynh-Feldt adjustments were used when sphericity could not be assumed. Significant main effects that were qualified by significant interactions are not reported.

Rating latencies. Suppression-induced distraction was evaluated by computing mean rating latencies, trimmed in each cell of eight observations to exclude times beyond 2.5 SD. These means were submitted to a mixed-design analysis of variance (ANOVA) with a withinsubjects factor for Flanker Status (the three critical sets of negative homographs assigned to baseline, suppress, and new in the prior "experiment") and a between-subjects factor for 
Suppression Instructions (direct-suppression vs. substitution). The interaction was significant, $F(1.79,71.61)=5.54, M S E=28833, p=.008, \eta_{\mathrm{p}}{ }^{2}=.12$. A follow-up interaction of Instruction with the comparison of suppression flankers to the other negative homographs explained $69 \%$ of the variance in the interaction, $F(1,40)=7.89, M S E=24900, p=.008, \eta_{\mathrm{p}}^{2}=.17$. Figure 2 makes it clear that suppressed flankers delayed responding, but only in the direct-suppression condition.

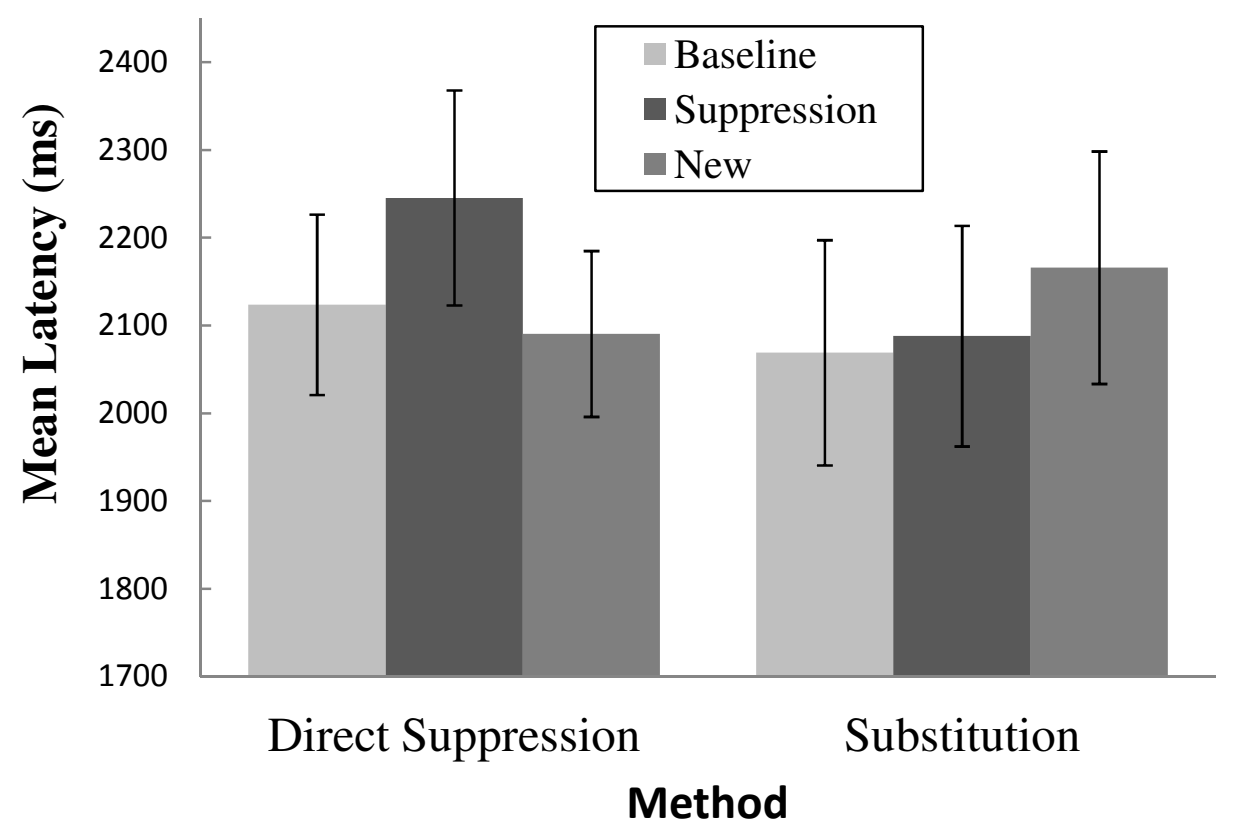

Figure 2. Mean latency to rate the emotional valence of the target when flanked by negative homographs that cued learned but not practiced responses (baseline), learned and suppressed responses (suppression), or no responses because they were encountered in the flanker task for the first time (new). Error bars represent standard errors of the mean.

When new negative homograph trials were omitted from the analysis of flanker RTs, the interaction of suppression method with the comparison between baseline and suppression flankers was only marginally significant, $F(1,40)=3.53, M S E=15609, p=.065, \eta_{\mathrm{p}}{ }^{2}=.08$. Clearly, some of the variance in the interaction can be attributed to the effect of method during trials with new negative flankers, an effect that itself was not significant, $p=.646$. However, the 
distraction effect (baseline vs. suppression flankers) was significant in the direct-suppression condition, $t(20)=3.03, p=.007, d=1.08, \mathrm{CI}=[38.0,205.5]$, and not in the substitution condition, $t(20)=0.52, p=.607, d=0.16, C I=[-96.3,57.7]$.

New homographs with negative meanings (used as new items in the previous analysis) did not retard responses to a greater extent than did similarly new homographs with neutral or positive meanings. An ANOVA with factors for Instruction and Flanker Valence (new negative, neutral, and positive) revealed nonsignificant effects; the smallest $p>.48$ and largest $\eta_{\mathrm{p}}{ }^{2}<.02$.

Valence ratings. Similar analyses were used to examine possible effects on ratings for the neutral targets. No evidence of distraction effects obtained when Flanker Status (baseline, suppress, new) was included as the within-subjects factor; smallest $p>.23$, largest $\eta_{\mathrm{p}}{ }^{2}<.04$. The overall mean rating was $5.4(1=$ very negative, $9=$ very positive $)$. When the instruction comparison was restricted to suppressed flankers, a nonsignificant difference obtained, $p>.20$. However, when the valence of the new flankers served as the within-subjects factor, an ANOVA performed on ratings for the neutral targets revealed a significant main effect of Flanker Valence, $F(2,80)=20.58, M S E=.233, p<.001, \eta_{\mathrm{p}}{ }^{2}=.34$. Means were 5.3 (new negative homographs), 5.6 (new neutral), and 6.0 (new positive). The interaction with Instruction was nonsignificant, $p=.78$ and $\eta_{\mathrm{p}}^{2}=.01$

Finally, we examined ratings for targets when flankers were neutral by including a within-subjects factor for the valence of the targets (negative, neutral, positive). Clearly, the participants were attending to the goal of the rating task; for the main effect of target valence, $F(2,80)=622.62, M S E=.529, p<.001, \eta_{\mathrm{p}}{ }^{2}=.94$. Means were 1.8 (negative targets), 5.6 (neutral), and 7.3 (new positive). The interaction with Instruction was again nonsignificant, $p=$ .53 and $\eta_{\mathrm{p}}^{2}=.01$ 
Suppression-induced forgetting. The percentages of response words correctly recalled during the final recall phase were analyzed in a mixed-design analysis of variance (ANOVA) with a within-subjects factor for Cue Status (baseline vs. suppression) and a between-subjects factor for Instruction (direct suppression vs. substitution). The extent of SIF depended on Instruction, $F(1,40)=4.08, M S E=164.44, p=.050, \eta_{\mathrm{p}}{ }^{2}=.09$ (Figure 3). Evidence for SIF obtained for participants who were asked to use benign substitutes, $\left(M_{\text {diff }}=17.3\right), t(20)=4.54$, $p<.001, \mathrm{CI}=[9.3,25.2]$, but not for those given instructions for direct suppression, $\left(M_{\mathrm{diff}}=\right.$ $6.0), t(20)=1.45, p=.16, \mathrm{CI}=[-2.6,14.5]$. Thus, SIF was obtained when an ostensibly unrelated task occurred during the retention interval, but only under conditions in which the cues exposed during that task might have been redefined by the substitutes used to aid suppression.

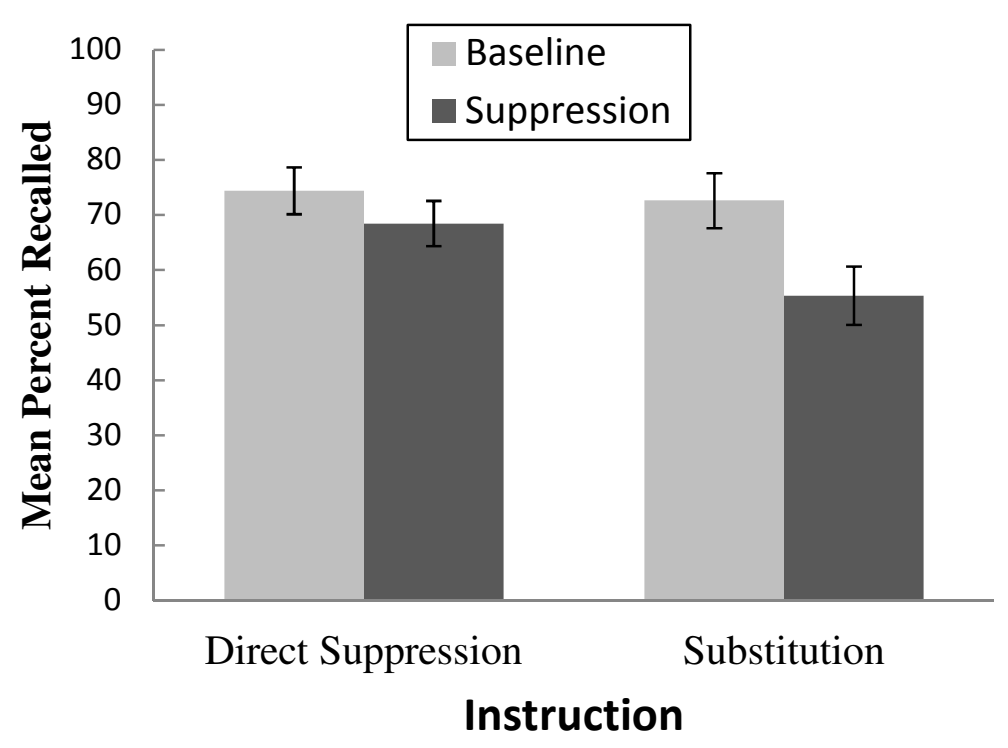

Figure 3. Mean percentage of response words recalled in each condition of Instruction and Cue Status. Response words practiced during the TNT phase were perfectly recalled by almost everyone. Error bars represent standard errors of the mean. 
In that same condition of substitute use, the extent of SIF was uncorrelated with suppression-induced distraction (defined in terms of latencies) in the intervening task, $r(19)=-.02, p=.92$. However, in the direct-suppression condition, the participants who were delayed to a greater extent by suppression cues in the flanker task also tended to produce the greater below-baseline forgetting in the recall task, $r(19)=.47, p=.03$.

Questionnaire measures. To measure noncompliance with suppression instructions, responses to three "strategy" items were averaged. A rating of 0 was meant to indicate that the participants never used a particular strategy to remind themselves of the suppressed words; 1 indicated rarely, 2 sometimes, 3 frequently, and 4 very frequently. The eight participants whose data were replaced scored greater than 1, on average (our cut-off). For the remaining participants, noncompliance did not depend on instruction, $M=0.48,95 \% \mathrm{CI}_{\mathrm{diff}}=[-0.10,0.33]$. The fourth item inquired about use of other thoughts and words to aid suppression attempts. Again, the two instructional conditions did not differ significantly, $M=2.57$ (direct suppression), $M=2.71$ (substitution), $C I_{\text {diff }}=[-0.46,0.75]$. They indicated that other thoughts were used somewhere between "sometimes" and "frequently." In the direct-suppression condition, this tendency was not significantly correlated with the measure of SIF, $r(19)=.33, p=.14$. A fifth item ("I found it very difficult to suppress thoughts of response words.") was rated as more frequently experienced by the direct-suppression participants $(M=1.76$ vs. 1.00 in the substitution condition), $t(40)=2.77, \mathrm{p}=.008, \mathrm{CI}=[0.21,1.32]$. Again, the report of difficulty in direct suppression was uncorrelated with SIF, $r(19)=-.09, p=.68$.

\section{Experiment 2}

We conducted Experiment 2 for two reasons. First, replicating the correlation between SIF and the distraction effect seemed particularly important to our attention explanation of the 
flanker effect. Second, we attempted to replicate the suppression effect on latencies with a streamlined rating procedure used during the word display. We glued dots to the $c, v, b, n$, and $m$ keys and asked participants to place the index and second finger of each hand on all keys but $b$.

\section{Method}

All aspects of Experiment-1 method were used, with the following changes. Only directsuppression instructions were used. With the constraint of equal cell sizes, 32 students were randomly assigned to combinations of a factor for the direction of the 5-point rating scale (positive to negative with the dominant hand on the positive side, or negative to positive with the dominant hand on the negative side) and a counterbalancing factor for rotating the critical sets across conditions of Cue/Flanker Status (baseline, suppression, new). The data from 8 participants were set aside- -6 according to the same noncompliance criterion as we used in Experiment 1, 1 because he could not stay awake, and 1 because English was the second language (missed during screening). Gender was evenly distributed across these six cells. And the number of cells led us to increase the sample size from 21 to 24 to achieve complete counterbalancing.

\section{Results}

The main dependent variables were the mean latency in ms to press the key corresponding to the valence rating and the percentage of words recalled on the final test. We performed dependent $t$ tests to examine differences between baseline and suppression cues on each measure. Latencies were longer when suppression cues served as flankers, $(M=2230$ vs. $2108), t(23)=2.98, p=.007, \mathrm{CI}=[37,207]$. A marginal SIF effect on cued recall was obtained, $(M=76 \%$ baseline vs. $68 \%$ suppression $), t(23)=2.00, p=.057, \mathrm{CI}=[-0.24,15.87]$. Moreover, the correlation between SIF and the distraction effect was again significant, $r(22)=.45, p=.03$. 
For the purpose of comparing more directly to Experiment-1 results, three repeatedmeasures ANOVAs were performed. Latencies did not significantly differ according to the three critical sets (baseline, suppression, and new), $F(2,46)=2.70, M S E=33199, p=.078, \eta_{\mathrm{p}}{ }^{2}=.10$. The mean latency for new negative homographs was 2172 , falling in the range between means for baseline and suppression flankers. The error term indicates larger variance than in Experiment 1 . However, $73 \%$ of the variability associated with Flanker Status was accounted for by the comparison between suppression cues and the other two conditions, $F(1,23)=3.85, M S E$ $=33983, p=.062, \eta_{\mathrm{p}}{ }^{2}=.14$. Again, the prior role of the critical-set flankers did not influence the ratings themselves, $F(2,44)=0.33, M S E=.115, p=.677, \eta_{\mathrm{p}}{ }^{2}=.02$; the overall $M=3.3$. (One student reversed the scale, so the data were excluded.) However, as in Experiment 1, the valence of the flankers influenced the ratings of the neutral targets, $F(2,44)=5.69, M S E=.987, p=$ $.006, \eta_{\mathrm{p}}{ }^{2}=.20$; The means were 3.3 for the new negative homographs, 3.5 for neutral homographs, and 3.6 for positive homographs (on a 5-pt scale).

Finally, means on the strategy questionnaire were similar to those in Experiment 1. Noncompliance was reported as low (among those whose data were not removed), $M=0.50$. Participants reported that they sometimes used other thoughts to aid suppression, even though we had heavily stressed the importance of not doing so, $M=1.92$. However, their reports were not significantly correlated with SIF, $r(22)=-.17, p=.42$. They sometimes found it very difficult to suppress thoughts of the response words, $\mathrm{M}=2.00$, although again this reported tendency was not associated with SIF, $r(22)=-.10, p=.63$.

\section{Discussion}

In two experiments, we found evidence for suppression-induced distraction as participants judged the emotional valence of neutral targets. Cues initially associated with 
negative responses later appeared in a presumably unrelated task as unimportant flankers surrounding the judged targets. In the interim think/no-think phase (after learning but before the flanker task), these cues were either not displayed (baseline) or they appeared multiple times to signal the suppression of their associates. When the suppression trials had been accomplished by stopping all thoughts (or so we instructed), subsequent valence judgments were delayed, compared to trials with baseline flankers. Although direct-suppression did not produce belowbaseline recall as has been shown in other experiments (e.g., Benoit \& Anderson, 2012), success in SIF was correlated with distraction in the judgment task. Moreover, the correlation was replicated in a second experiment with a different version of the judgment task. Thus, the best candidate mechanism for the distraction effect appears to be well-practiced thought stopping in the context of focused attention on the cues. Suppression aided by substitutes produced large decrements in recall of the negative response words, but the cues failed to interfere during the flanker task, even though they had been presented just as recently and frequently as in the directsuppression condition and even though visual focus during suppression was equally emphasized.

Several features of these experiments invite further explorations of the effects. First and perhaps most obviously, to reflect the fact that unwanted memories are generally negative memories, the responses that were suppressed in these and other related experiments were all emotionally negative (e.g., Hertel et al., 2012; Joormann, Hertel, Le Moult, \& Gotlib, 2009). However, it seems important to know if cues for suppressing positive memories (for the purpose of focusing on the mundane, for example) would engender similar or even greater distraction. In both of our experiments, positive and negative homographs biased the valence ratings of neutral targets to similar degrees, and their suppression might equally well cause distraction. 
A related feature of our work was the use of homographs, chosen so that a reconceptualization of the cue is afforded by learning benign substitutes. If the flanker effect is due in part to the meaning of the flankers being similar to their earlier negative meaning, then change in meaning should play an important role. Change in meaning via substitution can be evaluated by examining whether target ratings were affected by substitition (as they were in our subsidiary comparison across the valence of new flankers), but they were not. Compared both to baseline and new negative homographs in the substitution condition and to suppression cues in the direct-suppression condition, suppression cues for substitutes did not incur more positive judgments of the targets they surrounded. The case for redefinition therefore has not been made in this experiment (cf. Hertel et al., 2012). Regardless, we acknowledge that our use of homographs constrains the effects we report; the results might not generalize to cues with stable meanings. Emotional ambiguity is the exception in experiments with verbal materials, even though it might be ubiquitous in every-day situations.

Other issues raised by these experiments concern whether these distraction effects rely on the use of emotional materials or even the act of suppression itself. Perhaps cues for nonemotional memories would produce the same effect, although we cannot imagine why such memories would be deliberately suppressed. And indeed the act of concentrating intently on a word for whatever purpose might turn it into a distractor on a subsequent task. Our interest, however, was confined to the contributory effects of suppression to distraction, not its necessity.

Finally, we call attention to the evidence for SIF, believing that the effect has rarely been documented following an intervening task that displayed all cues in the context of an unrelated task. At traditional levels of significance, below-baseline forgetting occurred in the substitution condition of Experiment 1. It might have been slightly inflated by no requirement to produce a 
second word in those few cases when the substitute had been recalled alone, but this caveat holds for immediate substitution effects as well. In the direct-suppression condition of both experiments SIF could possibly have been reduced by occasional retrieval of the response word during the approximately $2 \mathrm{~s}$ it took to make the rating in the flanker task; such retrieval would arguably be less likely in the substitution condition. Yet, it also seems unlikely in Experiment 2, where an SIF effect of $8 \%$ was obtained following direct-suppression, and the effect was statistically significant on the basis of one-tailed hypothesis testing. There are other minor considerations that lead us to emphasize the SIF effects, such as the realization that even occasional retrieval of the response word on suppression trials should elevate recall above baseline levels (see Karpicke \& Roediger, 2008). Also, forgetting in the TNT paradigm is more difficult to achieve when the cues and response words are meaningfully related, as they are in these experiments (Hertel \& Mahan, 2008). Regardless of such difficulty, however, the individuals who achieved forgetting following instructions for direct suppression also tended to be the individuals who were bothered by the cues appearing in a different context. The moral of the story, therefore, is that there is a cost as well as a benefit to direct suppression. The cost might best be characterized as transfer of training the thought stopping procedure formerly practiced in a deliberate manner and now appearing as brief mind-blanking interludes when cues for unwanted thoughts are encountered. 


\section{References}

Anderson, M. C., \& Green, C. (2001). Suppressing unwanted memories by executive control. Nature, 410, 366-369.

Anderson, M. C., \& Hanslmayr, S. (2014). Neural mechanisms of motivated forgetting. Trends in Cognitive Science, 18, 279-292.

Anderson, M. C., \& Huddleston, E. (2012). Towards a cognitive and neurobiological model of motivated forgetting. In R. F. Belli (Ed.), True and false recovered memories: Toward a reconciliation of the debate (Nebraska Symposium on Motivation, Vol. 58, pp. 53-120). New York: Springer.

Anderson, M. C., \& Levy, B. J. (2009). Suppressing unwanted memories. Association for Psychological Science, 18, 189-194.

Anderson, M.C., Ochsner, K., Kuhl, B., Cooper, J., Robertson, E., Gabrieli, S.W., et al. (2004). Neural systems underlying the suppression of unwanted memories. Science, 303, 232235.

Beck, A. T., Steer, R. A., \& Brown, G. K. (1996). Manual for the Beck Depression Inventory-II. San Antonio, TX: Psychological Corporation.

Benoit, R. G., \& Anderson, M. C. (2012). Opposing mechanisms support the voluntary forgetting of unwanted memories. Neuron, 76, 450-460.

Bjork, R. A. (1989). Retrieval inhibition as an adaptive mechanism in human memory. In H. L. Roediger \& F. I. M. Craik (Eds.), Varieties of memory and consciousness: Essays in honour of Endel Tulving (pp. 309-330). Hillsdale, NJ: Erlbaum. 
Bradley, M. M., \& Lang, P. J. (1999). Affective norms for English words (ANEW): Instruction manual and affective ratings. Technical Report C-1, The Center for Research in Psychophysiology, University of Florida.

Depue, B. E., Curran, T., \& Banich, M. T. (2007). Prefrontal regions orchestrate suppression of emotional memories via a two-phase process. Science, 317, 215-219. DOI: $10.1126 /$ science. 1139560

Eriksen, B. A., \& Eriksen, C. W. (1974). Effects of noise letters upon identification of a target letter in a non- search task. Perception and Psychophysics 16, 143-149. doi: $10.3758 / \mathrm{bf} 03203267$

Gagnepain, P., Henson, R. N., \& Anderson, M. (2014). Suppressing unwanted memories reduces their unconscious influence via targeted cortical inhibition. Proceedings of the National Academy of Sciences of the United States of America. Published online doi: $10.1073 /$ pnas. 1311468111

Hertel, P. T., \& Calcaterra, G. (2005). International forgetting benefits from though substitution. Psychonomic Bulletin \& Review, 12(3), 484-489.

Hertel, P.T., \& Gerstle, M. (2003). Depressive deficits in forgetting. Psychological Science, 14, 573-578.

Hertel, P. T., Large, D., Stück, E. D., \& Levy, A. (2012). Suppression-induced forgetting on a free-association test. Memory, 20, 100-109.

Hertel, P. T., \& Mahan, A. (2008). Depression-related differences in learning and forgetting responses to unrelated cues. Acta Psychologica, 127, 636-644.

Holmes, E. A., Moulds, M. L., \& Kavanagh, D. (2007). Memory suppression in PTSD treatment [letter to the editor]. Science, 318, 1722. 
Joormann, J., Hertel, P. T., LeMoult, J., \& Gotlib, I. H. (2009). Training forgetting of negative material in depression. Journal of Abnormal Psychology, 118, 34-43.

Karpicke, J. D., \& Roediger, H. L. (2008). The critical importance of retrieval for learning. Science, 319, 966-968.

Kim, K., \& Yi, D.-J. (2013). Out of mind, out of sight: perceptual consequences of memory suppression. Psychological Science, 24, 569-574.

Kucera, H., \& Francis, W. N. (1967). Computational analysis of present-day American English. Providence, RI: Brown University Press.

Nelson, D. L., McEvoy, C. L., \& Schreiber, T. A. (1998). The University of South Florida word association, rhyme, and word fragment norms. Retrieved from http://www.usf.edu/FreeAssociation/

Nolen-Hoeksema, S., \& Morrow, J. (1991). A prospective study of depression and posttraumatic stress symptoms after a natural disaster: The 1989 Loma Prieta earthquake. Journal of Personality and Social Psychology, 61, 115-121.

Stephens, E., Braid, A., \& Hertel, P. T. (2013). Suppression-induced reductions in the specificity of autobiographical memories. Clinical Psychological Science, 1, 163-169.

Wegner, D. M. (1994). Ironic processes of mental control. Psychological Review, 101, 34-52. 


\begin{abstract}
Author Contributions
PH developed the idea, designed the experiments, and wrote the manuscript, all with contributions from JH. JH constructed materials and collected data. Each author analyzed the data.
\end{abstract}

\title{
Acknowledgments and Funding
}

The authors thank Colleen Kelley and Nilly Mor for discussion of the issues, Nupur Agrawal and Sara Touchstone for scoring and, especially, Alexandra Barrionuevo for assistance in programming, material construction, and data collection. AB was supported by a grant from the United States/Israel Binational Science Foundation (BSF 2011267). 